\title{
The Comparison Of Two Different Concentrations Of Ketofol For Pediatric Procedural Sedation In Circumcision Surgeries
}

\author{
Vuslat Muslu Erdem¹, Turgut Donmez², Duygu Ayfer Erdem¹, Ekrem Yadigaroglu³
}

${ }^{1}$ Department of Anesthesiology, Lutfiye Nuri Burat State Hospital, Istanbul, Turkey

${ }^{2}$ General Surgery, Bakırkoy Dr. Sadi Konuk Research And Training Hospital, Istanbul, Turkey

${ }^{3}$ Department of Urology, Lutfiye Nuri Burat State Hospital, Istanbul, Turkey

\section{INTRODUCTION}

Since combination of ketamine and propofol lacks respiration related problems and opioid-associated adverse effects, such as respiratory depression, postoperative nausea and vomiting, compared to other anesthetic agents, ketofol has been increasingly used for anesthesia for children.

\section{OBJECTIVE}

The aim of our retrospective study was to evaluate the intraoperative sedation quality and side effects of two different concentrations of ketofol, $1: 2(0.5 \mathrm{mg} / \mathrm{kg}-1.0 \mathrm{mg} / \mathrm{kg}$ ) ketamine-propofol versus $1: 3(0.5 \mathrm{mg} / \mathrm{kg}-1.5 \mathrm{mg} / \mathrm{kg})$ ketamine-propofol, on children who underwent circumcision surgery.

\section{METHODS}

We enrolled 102 patients (American Society of Anesthesiologists[ASA] class I-II) who received either 1:2(0.5mg/kg$1.0 \mathrm{mg} / \mathrm{kg}$ ) ketamine-propofol (Group A, $\mathrm{n}=50$ ) or $1: 3(0.5 \mathrm{mg} / \mathrm{kg}-1.5 \mathrm{mg} / \mathrm{kg}$ ) ketamine-propofol (Group B, $\mathrm{n}=52$ ) for general anesthesia from June 2017 to December 2017 . Sedation quality was assessed using the Richmond Agitation and Sedation Scale (RASS).

\section{RESULTS}

\section{The Richmond Agitation and Sedation Scale (RASS)}

\begin{tabular}{c|l|l} 
Score & \multicolumn{1}{|c|}{ Term } & \multicolumn{1}{c}{ Description } \\
\hline+4 & Combative & $\begin{array}{l}\text { Overtly combative or violent; immediate } \\
\text { danger to staff }\end{array}$ \\
\hline+3 & $\begin{array}{l}\text { Very } \\
\text { agitated }\end{array}$ & $\begin{array}{l}\text { Pulls on or removes tube(s) or catheter(s) } \\
\text { or has aggressive behavior toward staff }\end{array}$ \\
\hline+2 & Agitated & $\begin{array}{l}\text { Frequent nonpurposeful movement or } \\
\text { patient-ventilator dyssynchrony }\end{array}$ \\
\hline
\end{tabular}

\begin{tabular}{ll|l}
$\mathbf{1 1}$ & Restless & $\begin{array}{l}\text { Anxious or apprehensive but movements } \\
\text { not aggressive or vigorous }\end{array}$ \\
\hline $\mathbf{0}$ & $\begin{array}{l}\text { Alert and } \\
\text { calm }\end{array}$ & $\begin{array}{l}\text { Spontaneously pays attention to caregiver } \\
\text { Not fully alert, but has sustained (more }\end{array}$ \\
\hline $\mathbf{- 1}$ & $\begin{array}{l}\text { Drowsy } \\
\text { than } 10 \text { seconds) awakening, with eye } \\
\text { contact, to voice }\end{array}$ \\
\hline $\mathbf{- 3}$ & $\begin{array}{l}\text { Light } \\
\text { sedation } \\
\text { sedation }\end{array}$ & $\begin{array}{l}\text { Briefly (less than } 10 \text { seconds) awakens with } \\
\text { eye contact to voice }\end{array}$ \\
\hline-4 & $\begin{array}{l}\text { Any movement (but no eye contact) to } \\
\text { voice }\end{array}$ \\
\hline sedation & $\begin{array}{l}\text { No response to voice, but any movement to } \\
\text { physical stimulation }\end{array}$ \\
\hline-5 & $\begin{array}{l}\text { Unarousabl } \\
\text { e }\end{array}$ & No response to voice or physical stimulation \\
\hline
\end{tabular}

Sedation scores (RASS) at 5, 10, 15 and 20 min during operation.

\begin{tabular}{|l|c|c|c|} 
& $\begin{array}{c}\text { Group A (1:2) } \\
\mathbf{n}=\mathbf{5 0}\end{array}$ & $\begin{array}{c}\text { Group B(1:3) } \\
\mathbf{n = 5 2}\end{array}$ & P-Value \\
\hline $\mathbf{5} \mathrm{min}$ & -4 & -5 & $<0.001$ \\
\hline $10 \mathrm{~min}$ & -4 & -4 & $<0.001$ \\
\hline $15 \mathrm{~min}$ & -3 & -4 & 0.08 \\
\hline $\mathbf{2 0} \mathrm{min}$ & -2 & -2 & $<0.001$ \\
\hline
\end{tabular}

\begin{tabular}{l|c|c|}
\hline $\begin{array}{l}\text { Postoperative adverse } \\
\text { events }\end{array}$ & $\begin{array}{c}\text { Group A(1:2) } \\
n=\mathbf{5 0}\end{array}$ & $\begin{array}{c}\text { Group B(1:3) } \\
n=52\end{array}$ \\
\hline Vomiting & 1 & 0 \\
\hline Hallucination & 3 & 0 \\
\hline Respiratory depresyon & 2 & 3 \\
\hline
\end{tabular}

Group A (1:2)

\section{Number of Patients}

\section{CONCLUSION}

Our study showed that $1: 3(0.5 \mathrm{mg} / \mathrm{kg}-1.5 \mathrm{mg} / \mathrm{kg})$ ketamine-propofol mix provided better intraoperative sedation quality with fewer side effects compared to $1: 2(0.5 \mathrm{mg} / \mathrm{kg}-1.0 \mathrm{mg} / \mathrm{kg}) \mathrm{ketamine-propofol} \mathrm{mix.}$

In Group B(1:3 ketamine-propofol), all of the patients exhibited satisfactory sedation for the duration of 30 min after anesthesia induction. Repeat dose of ketofol was not necessary. However, in Group A(1:2 ketamine-propofol), repeat doses of ketofol were needed for some patients. 